\title{
TRANSVERSE MYELITIS IN A MYASTHENIA GRAVIS PATIENT. A CASE PRESENTATION
}

\author{
Ruxanda Dana Chirileanu, ${ }^{1,2}$, Mihaela Simu ${ }^{1,2}$, Cecilia Rosca ${ }^{1,2}$, Raluca Tudor ${ }^{1,2}$, \\ Patricia Jurca ${ }^{2}$ \\ 1 "Victor Babes" University of Medicine and Pharmacy, Timisoara \\ ${ }^{2}$ Department of Neurology, Emergency County Hospital of Timisoara
}

\begin{abstract}
Myasthenia gravis (MG) is an autoimmune disease caused by the presence of immunoglobulin $\mathrm{G}(\lg \mathrm{g}) 1$ and $\lg \mathrm{G} 3$ complement activating antibodies against the nicotinic acetylcholine receptor, which affects the neuromuscular junction leading to fluctuating muscle weakness due to impaired neuromuscular transmition. The association of MG and demyelinating diseases is rare, but it has been described before and it could be part of an unspecific immune activation, due to genetic susceptibility or it could just happen randomly. Demyelinating diseases (DD) in MG patients can occur as monophasic events (myelitis, optic neuritis, acute disseminated encephalomyelitis) or recurrent diseases (multiple sclerosis, recurrent transverse myelitis) and since the incidence of DD is higher in MG patients than in general population this association could be part of an autoimune syndrome or genetically induced. We present the case of a 30 year old woman who presented with an unspecific onset of MG and after 5 months was readmitted to our unit with transverse myelitis (with negative aquaporin 4 antibodies) which regressed after 5 days of combined intravenous corticotherapy and immunoglobulin treatment.
\end{abstract}

Keywords: myastenia gravis, transverse myelitis, aquaporin 4 antibodies, autoimune syndrome

\section{INTRODUCTION}

Myastenia gravis is caused by autoantibodies specific for the human nicotinic acethylcholine receptor which are present in about $85 \%$ of $M G$ patients. MG is a disease affecting mostly women between 20 and 40 years old and mostly men between 60 and 80 years old. The seronegative forms could be attributed to antibodies targeting muscle specific kinase or other proteins in the post synaptic muscle membrane.

Acute transverse myelitis is one of the causes of acute transverse myelopathy. Acute transverse myelopathy can have different causes: compression, vascular, neoplasic, paraneoplasic and inflammatory disorders (demyelination, infections herpes zoster, herpes simplex virus and other inflammatory disorders - like lupus erythematosus). Demyelinating disorders such as multiple sclerosis
(MS), neuromyelitis optica spectrum disorder (NMOSD) and idiopathic transvers myelitis are the most common causes of acute transverse myelitis. In MS patients the magnetic resonance imaging (MRI) of the spinal cord shows lesions in less than 2 spinal cord segments, the disease may be asymptomatic and oligoclonal bands are present in the cerebrospinal fluid. The NMOSD diagnostic criteria for NMOSD without aquaporin $4 \mathrm{IgG}$ are:

1. at least 2 core clinical characteristics as a result of one or more clinical attacks - at least 1 core clinical characteristic must be optic neuritis, acute myelitis with longitudinally extensive transverse myelitis lesions or area postrema syndrome, dissemination in space for the clinical characteristics, supportive MRI characteristics;

2. negative aquaporin $4 \mathrm{IgG}$;

3. exclusion of other diagnoses. 
The core clinical characteristics are: optic neuritis, acute myelitis, area postrema syndrome, acute brainstem syndrome, symptomatic narcolepsy or acute diencephalic clinical syndrome with MRI lesions and symptomatic cerebral syndrome with brain lesions. NMOSD are divided into NMOSD with or without aquaporin $4 \mathrm{IgG}$, most of the patients being seropositive. There are no clinical pathognomonic presentations of NMOSD, but a single clinical manifestation in seronegative patients excludes NMOSD. The criteria are used to ease diagnosis when dealing with idiopathic transverse myelitis, idiopathic optic neuritis or atyphical MS. Inclusion criteria for idiopathic transverse myelitis are: sensory, autonomic or motor spinal cord dysfunction, bilateral symptoms and clearly defined sensory level, exclusion of compressive ethiology, inflammation within the spinal cord and progression of the symptoms since onset.

The association of MG to demyelinating diseases is rare but it has been reported before, especially the association of MG and NMOSD; because of the rarity of the 2 diseases their association is not considered coincidental, generally the MG symptoms are mild, but the NMOSD symptoms tend to follow more aggresive presentations. The fact that both aquaporin $4 \mathrm{IgG}$ and the antibodies against the nicotinic acetylcholine receptor are predominantly IgG1 complement activating could be involved in the diseases association. The association usually occurs in patients with controlled MG symptoms; patients severly affected may be on more aggresive treatment that could prevent NMOSD clinical symptoms, so screening for aquaporin $4 \mathrm{IgG}$ in patients with $M G$ is recommended when they present with inflammatory demyelinating conditions of the central nervous system. In seronegative patients diagnoses such as MS, NMOSD without aquaporin 4 $\mathrm{IgG}$, acute diseminated encefalomyelitis, lupus erythematosus, or idiopathic transverse myelitis should be considered.

\section{CASE REPORT}

We present the case of a 30-year-old woman who was first admitted in our clinic in march 2015 with fatigue, paresthesias and muscular cramps in both of her legs which made it difficult for her to walk. Symptoms started a month before presentation, lasted all day long and apparently improved after resting. She had no autoimune diseases in her family, no other conditions and wasn't on any treatment or drugs. On neurological examination she had a slightly antalgic gait, other than that no neu- rological abnormalities were revealed, general clinical examination was normal, laboratory tests were in normal rates, abdominal ultrasound showed no pathological changes, chest x-ray was normal, brain CT scan was normal, spinal cord MRI showed minimal arthritis of the spinal apophysis at L3-L4 level, moderate fluid level in Douglas space, minimal discal protrusion at C5-C6 level without radicular compresion, and no spinal cord modifications. Neuromuscular electromyogram showed neurogenic modifications and nerve conduction velocity was within normal rates. Lumbar puncture was performed and cerebrospinal fluid analysis showed no abnormalities. Gynecological examination showed no abnormalities and psychological evaluation showed modifications of depression and anxiety. Since admission she was started on oral treatment with nonsteroidal anti-inflammatory drugs and antidepressants and the initial symptoms seemed to regress, although she started to develop fatigue and paresthesias in the facial muscles bilateral after 1 week. Screening for antibodies against the nicotinic acetylcholine receptor was performed and the patient was diagnosed with discopathy and anxiety disorder until the results came. Since antibodies were positive, the patient was diagnosed with MG and started on 3 tablets of Mestinon per day. Under this therapy all of her symptoms improved and she was released stable without any complaints after 1 week of treatment with Mestinon.

In august 2015 the patient was readmitted to our unit, this time for Brown Sequard hemicord syndrome on the right, with ipsilateral pyramidal weakness, loss of posterior column function on the right and contralateral spinothalamic loss. The symptoms had subacute onset during a period of about a week. Her MG symptom was mild fatigue, but she had a difficult gait with unilateral support, right leg paresis, Babinski sign was present on the right, she had increased osteotendinous reflexes on the right and loss of proprioceptive and vibratory function on the right and thermoalgesic hypoesthesia on the left hemibody with T4 sensory level. General clinical examination was normal, laboratory tests were in normal rates, chest $\mathrm{x}$-ray was normal, brain MRI was normal, and spinal cord MRI showed minimal discal protrusion at C5-C6 level, no cord compression and demyelination from $\mathrm{T} 1$ to $\mathrm{T} 6$ central and bilateral at the proximal level and only on the right at the distal level, modifications that were conclusive with transverse myelitis. Aquaporin $4 \mathrm{IgG}$ were negative, ophthalmological exam was normal and both psychological and psychiatric evaluations showed somatoform-anxiety disorder. The patient 


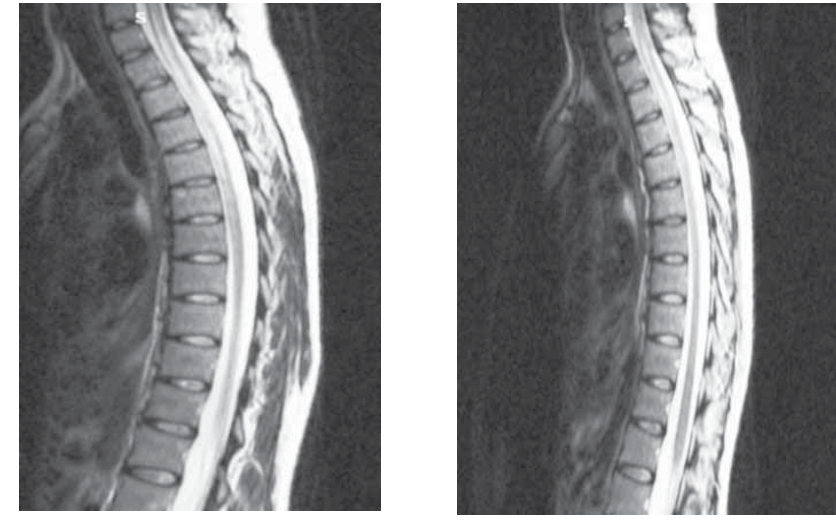

FIGURE 1. Spinal cord MRI of the patient showing demyelination

was started on intravenous corticotherapy and oral treatment with an antidepressant since admission. After 4 days of corticotherapy the symptoms showed no improvement and intravenous therapy with immunoglobulin was added. After 5 days of immunoglobulin the paresis went from $1 / 5$ to $+3 / 5$ in clinostatism, she had no more objective sensory deficiencies, only slightly paresthesias in her right hemibody and she could walk with a cane. She was released with indications of physiotherapy and treatment with mestinon 3 tablets per day and antidepressant therapy. At her 2 months follow up she had no relapses, her gait improved significantly, although she still used the cane and still had mild paresthesias in her righr hemibody. At her 4 months follow up she had a normal gait, no more paresthesias and still no relapses or ocular symptoms.

\section{DISCUSSIONS}

This case is interesting because MG usually associates with transverse myelitis as part of NMOSD, but in this case the patient didn't seem to meet NMOSD without aquaporin $4 \mathrm{IgG}$ criteria. She had only 1 core clinical characteristic, even if that was transverse myelitis, the spinal cord MRI was conclusive with the demyelinating disease and no other diagnoses were found (no oligoclonal bands in the cerebrospinal fluid, no inflammatory markers were found, no IgG for herpes virus, and tests for HIV virus and syphilis were negative). She rather met idiopathic transverse myelitis criteria since she had: sensory and motor spinal cord dysfunction, bilateral symptoms, clearly defined sensory level, exclusion of compressive ethiology, inflammation within the spinal cord was present at the MRI and she manifested a progression of the symptoms since onset. She didn't have a classical onset of MG neither, since her disease started with uncharacte- ristic symptoms like parasthesias and muscular cramps. She also had somatoform-anxiety symptoms which tricked us into diagnosing her with anxiety disorder and discopathy before we discovered that her antibodies against the nicotinic acetylcholine receptor levels were high. This disorder also led to the exageration of the myelopathy symptoms, especially the motor disfunction.

Demyelinating diseases associate in rare cases with MG, but when they do it could be part of an autoimmune syndrome or it could be due to genetic predisposition. NMOSD should be first considered in patients with MG who present with inflammatory demyelinating symptoms and when aquaporin 4 IgG are negative, additional tests should be performed to search for other inflammatory disorders or viral infections. There have been reported cases of patients with NMOSD symptoms without aquaporin $4 \mathrm{IgG}$, who had detectable serum myelin oligodendrocyte glycoprotein antibodies, suggesting that there could be other antibodies involved in the pathophysiology of NMOSD without aquaporin $4 \mathrm{IgG}$. Also, occasionally, patients without aquaporin $4 \mathrm{IgG}$ and symptoms meeting NMOSD criteria are found later seropositive; this happens usually in the case of technical errors while testing, in case of relapse, or when patients are tested while plasma exchange or immunosuppresive drugs are used. Our patient had only 1 core clinical manifestation out of the 5 described in NMOSD, so when she tested negative for aquaporin 4 antiboodies, we didn't test for other antibodies that have been associated with NMOSD in literature, because she didn't meet NMOSD criteria in the first place. Although she was tested while she was on corticotherapy, false negative results have only been described in patients using immunosuppresive drugs.

Regarding treatment, most NMOSD patients respond to steroids and plasmapheresis in acute phase. Our patient didn't show any improvements after 4 days of dexamethasone alone, immunoglobulin had to be added to treatment in order to show clinical response.

\section{CONCLUSIONS}

We presented a case of MG associated with idiopathic transverse myelitis in a 30-year-old woman with uncharacteristic onset of MG. Usually MG is associated with transverse myelitis as part of NMOSD, but in this case the patient didn't meet the criteria and no other cause of myelitis was found. Demyelinating diseases can occur in $\mathrm{MG}$ as part of an autoimmune syndrome, randomly or due to ge- 
netic predisposition. When aquaporin $4 \mathrm{IgG}$ are negative in patients with $\mathrm{MG}$ and inflammatory demyelinating symptoms which don't meet NMOSD criteria, additional testing should be per- formed, false-negative results excluded, and if no relapses or seroconversion are later identified, only then the demyelinating disease can be considered as idiopathic.

\section{REFERENCES}

1. Anu Jacob, Brian G. Wein Shenker; An Approach to the Diahnosis of Acute Transverse Myelitis; Seminars in neurology volume 28 number 12008.

2. Wingerchuk D.M., Banwell B., Bennett J.L., Cabre P., Carroll W., Chitnis T., de Seze J., Fujihara K., Greenberg B., Jacob A., Jarius S., Lana-Peixoto M., Levy M., Simon J.H., Tenembaum S., Traboulsee A.L., Waters P., Wellik K.E., Weinshenker B.G. International consensus diagnostic criteria for neuromyelitis optica spectrum disorders; Neurology. 2015 Jul 14; 85(2):177-89. doi: 10.1212/WNL.0000000000001729. Epub 2015 Jun 19.

3. Denis Bernardi Bichuetti, Tatiane Martins de Barros, Enedina Maria Lobato Oliveira, Marcelo Annes, Alberto Alain Gabbai; Demyelinating disease in patients with myasthenia gravis; Arq. Neuro-Psiquiatr. vol.66 no.1 São Paulo Mar. 2008.

4. Spillane J., Christofi G., Sidle K.C., Kullmann D.M., Howard R.S.; Myasthenia gravis and neuromyelitis opica: A causal link; Mult Scler Relat Disord. 2013 Jul;2(3):233-7. doi: 10.1016/j.msard.2013.01.003. Epub 2013 Feb 14.

5. M.I. Leite, E. Coutinho, M. Lana-Peixoto, S. Apostolos, P. Waters, D. Sato, L. Melamud, M. Marta, A. Graham, J. Spillane, A.M. Villa, D. Callegaro, E. Santos,A. Martins da Silva, S. Jarius, R. Howard, I. Nakashima, G. Giovannoni, C. Buckley, D. Hilton-Jones,

A. Vincent, J. Palace; Myasthenia gravis and neuromyelitis optica spectrum disorder A multicenter study of 16 patients; Neurology 2012 May 15; 78(20): 1601-1607.

6. Elena Sherman, May H. Han; Acute and Chronic Management of Neuromyelitis Optica Spectrum Disorder; Curr Treat Options Neurol. 2015; 17(11):48. 